\title{
CONTINUITY OF LIE DERIVATIONS ON BANACH ALGEBRAS
}

\author{
by M. I. BERENGUER and A. R. VILLENA
}

(Received 14th January 1997)

\begin{abstract}
The separating subspace of any Lie derivation on a semisimple Banach algebra $A$ is contained in the centre
\end{abstract} of $A$.

1991 Mathematics subject classification: 46H40, 17B40.

The Lie structure induced on a Banach algebra by the bracket $[a, b]=a b-b a$ is of lively interest for their intimate connections with the geometry of manifolds modeled on Banach spaces. Many mathematics have studied Lie derivations on associative rings $[1,5]$ and Lie derivations on some Banach algebras $[2,7,8]$.

A Lie derivation of a Banach algebra $A$ is a linear map $D$ from $A$ into itself satisfying $D([a, b])=[D(a), b]+[a, D(b)]$ for all $a, b \in A$. In this paper we study the continuity of a Lie derivation $D$ on an arbitrary semisimple Banach algebra $A$. We measure the continuity of $D$ by considering its separating subspace, which is defined as the subspace $S(D)$ of those elements $a \in A$ for which there is a sequence $\left\{a_{n}\right\}$ in $A$ satisfying $\lim a_{n}=0$ and $\lim D\left(a_{n}\right)=a . \mathcal{S}(D)$ is easily checked to be a Lie ideal of $A$ and the closed graph theorem shows that $D$ is continuous if, and only if, $\mathcal{S}(D)=0$.

Until further notice we assume that $A$ is a unital semisimple complex Banach algebra and $D$ stands for a Lie derivation of $A$. Let $\mathcal{Z}(A)$ denote the centre of $A$. For each $a \in A$ let ad $a$ denote the continuous linear operator $\operatorname{ad} a(b)=[a, b]$ from $A$ into itself. If $P$ is a closed ideal of $A$ we will denote by $Q_{P}$ the quotient map from $A$ onto $A / P$.

The next important result was essentially stated by $\mathbf{M}$. P. Thomas and illustrate the typical sliding hump argument.

Lemma 1 [10, Proposition 1.3]. Let $X$ and $Y$ be Banach spaces, $\left\{T_{n}\right\}$ a sequence of continuous linear operators from $X$ into itself, and let $\left\{R_{n}\right\}$ be a sequence of continuous linear operators from $Y$ into Banach spaces $Y_{n}$. If $F$ is a linear operator from $X$ into $Y$ such that $R_{n} F T_{1} \cdots T_{m}$ is continuous for $m>n$, then $R_{n} F T_{1} \cdots T_{n}$ is continuous for sufficiently large $n$. 
Lemma 2. If $P$ is a primitive ideal in $A$ of infinite codimension, then $[A, \mathcal{S}(D)] \subset P$.

Proof. Let us first observe that the extended centroid of $A / P$ is $\mathbb{C}$ (see [6, Theorem 12]) and does not satisfy the standard polynomial identity $S_{4}$ (see [9, Theorem 7.1.14]).

We claim that there exist $\lambda \in \mathbb{C}$, a linear functional $\mu$ on $A$, and a functional $\nu$ on $A$ such that

$$
\left(D\left(a^{2}\right)-(D a) a-a(D a)\right)-\left(\lambda a^{2}+\mu(a) a+v(a)\right) \in P
$$

for all $a \in A$. Indeed, for every $a \in A$, we have

$$
0=D\left(\left[a^{2}, a\right]\right)=\left[D a^{2}, a\right]+\left[a^{2}, D a\right]=\left[D a^{2}-(D a) a-a(D a), a\right] .
$$

Consequently, the map $q$ defined on $A$ by $q(a)=D a^{2}-(D a) a-a(D a)$ is a commuting trace of the bilinear map $B(a, b)=D(a b)-(D a) b-a(D b)$ on $A \times A$. The map $q$ can be handled in the same way as in the proof of Theorem 1 in [1], the only difference being in the application of [1, Lemmas 1 and 2] to $A / P$ instead of $A$.

We can now proceed as in the proof of [1, Theorem 4] in order to prove that the map $d$ defined on $A$ by $d(a)=D(a)+\lambda a+\frac{1}{2} \mu(a)$ satisfies

$$
d(a b)-(d(a) b+a d(b)) \in P
$$

for all $a, b \in A$ and therefore $Q_{P} d$ is a derivation from $A$ to $A / P$. Indeed, the identity (4) in that proof becomes

$$
d(a b+b a)-(d(a) b+d(b) a+a d(b)+b d(a)+\rho(a, b)) \in P
$$

for all $a, b \in A$, for a suitable symmetric bilinear functional $\rho$ on $A \times A$. The identity (7) now becomes

$$
\begin{gathered}
\rho(a, a)([a b, c]+[b a, c])-\rho(a, b)\left[a^{2}, c\right]-\rho\left(a, a^{2}\right)[b, c]+ \\
\left(2 \rho\left(a^{2}, b\right)-\rho(a, a b+b a)\right)[a, c] \in P
\end{gathered}
$$

for all $a, b, c \in A$. In particular $\rho(a, a)\left[a^{2}, c\right]-\rho\left(a, a^{2}\right)[a, c] \in P$ which gives $\rho(a, a)\left[\left[a^{2}, c\right]\right.$, $[a, c]] \in P$ for all $a, c \in A$. The arguments used in the proof of [1, Theorems 2 and 4] apply to this situation and it may be concluded that $\rho(a, b)=0$ for all $a, b \in A$. Consequently, $d(a \cdot b)-(d(a) \cdot b+a \cdot d(b)) \in P$ for all $a, b \in A$, where $a \cdot b=\frac{1}{2}(a b+b a)$. By the same method as at the end of the proof of [1, Theorem 4] we get the relation $[a, b] r(d(a b)-d(a) b-a d(b)) s[a, b] \in P$ for all $a, b, r, s \in A$, which yields the desired conclusion, since $A / P$ is not commutative.

Our next goal is to prove that $\mathcal{S}(d) \subset P$. To this end we set an infinite-dimensional complex irreducible Banach left $A$-module $X$ such that $P=\{a \in A: a X=0\}$. We apply the construction in [4, Theorem 2.2] to get sequences $\left\{a_{n}\right\}$ in $A$ and $\left\{x_{n}\right\}$ in $X$ such that 
$a_{n} \cdots a_{1} x_{n} \neq 0$ and $a_{n+1} a_{n} \cdots a_{1} x_{n}=0$ for all $n \in \mathbb{N}$. For $n \in \mathbb{N}$, we define the continuous linear operators $T_{n}(a)=a a_{n}$ from $A$ into itself, $R_{n}(a+P)=a x_{n}$ from $A / P$ into $X$, and $S_{n}(a+P)=a a_{n}+P$ from $A / P$ into itself. It is a simple matter to verify that $R_{n} Q_{P} d T_{1} \cdots T_{m}$ is continuous for $m>n$. Lemma 1 shows that $R_{n} Q_{P} d T_{1} \cdots T_{n}$ is continuous for some $n \in \mathbb{N}$. On the other hand, it is immediate that $Q_{P} d T_{1} \cdots T_{n}-S_{1} \cdots S_{n} Q_{P} d$ is continuous and therefore $R_{n} S_{1} \cdots S_{n} Q_{P} d$ is continuous. Consequently,

$$
0=R_{n} S_{1} \cdots S_{n} \mathcal{S}\left(Q_{P} d\right)=\mathcal{S}\left(Q_{P} d\right) a_{n} \cdots a_{1} x_{n}
$$

Since $\mathcal{S}\left(Q_{P} d\right)$ is easily seen to be a two-sided ideal of $A / P$, we see that

$$
\mathcal{S}\left(Q_{P} d\right) X=\mathcal{S}\left(Q_{P} d\right)(A / P) a_{n} \cdots a_{1} x_{n} \subset \mathcal{S}\left(Q_{P} d\right) a_{n} \cdots a_{1} x_{n}=0
$$

Hence $\mathcal{S}\left(Q_{P} d\right)=0$ and therefore $\mathcal{S}(d) \subset P$.

For every $a \in A$, we have adad $=\operatorname{ad} a D+\lambda \operatorname{ad} a$ and [9, Proposition 6.1.9(c)] shows that $\mathcal{S}(\operatorname{ad} a D)=\overline{\operatorname{ad} a(\mathcal{S}(D))}$ and $\mathcal{S}(\operatorname{adad})=\overline{\operatorname{ad} a(\mathcal{S}(d))}$. Therefore

$$
[a, \mathcal{S}(D)] \subset \overline{\operatorname{ad} a(\mathcal{S}(D))}=\mathcal{S}(\operatorname{ad} a D)=\mathcal{S}(\operatorname{ad} a d)=\overline{\operatorname{ad} a(\mathcal{S}(d))} \subset P
$$

for all $a \in P$.

Lemma 3. $\mathcal{S}(D) \subset \mathcal{Z}(A)$.

Proof. Suppose that the result fails. Then the set $\mathcal{P}$ of those primitive ideals $P$ of $A$ for which $[A, S(D)] \not \subset P$ is non empty. According to Lemma 2 each $P \in \mathcal{P}$ has finite codimension and therefore $A / P$ is simple. Moreover it is obvious that $\operatorname{dim} A / P>1$. On account of [3, Theorem 2 and Corollary 1] $Q_{P}(\mathcal{S}(D))=A / P$ for every $P \in \mathcal{P}$. Let $I_{0}$ denote the intersection of all the primitive ideals of $A$ for which $[S(D), A] \subset P$.

Take $P_{1} \in \mathcal{P}$. Since $I_{0} \not \subset P_{1}$ and $A / P_{1}$ is simple, it follows that $Q_{P_{1}}\left(I_{0}\right)=A / P_{1}$. Since $A / P_{1}$ is not commutative we can choose $a_{1} \in I_{0}$ such that $\left[a_{1}, A\right] \not \subset P_{1}$.

Assume that $P_{1}, \cdots, P_{n}$ and $a_{1}, \cdots, a_{n}$ have been chosen satisfying the following conditions:

(i) $P_{k} \in \mathcal{P}$,

(ii) $a_{k} \in I_{k-1}$, where $I_{k}=I_{0} \cap P_{1} \cap \cdots \cap P_{k}$,

(iii) $\operatorname{ad} a_{1} \cdots \operatorname{ad} a_{k}(A) \not \subset P_{k}$,

for $k=1, \cdots, n$. We claim that there exists $P_{n+1} \in \mathcal{P}$ such that $I_{n} \not \subset P_{n+1}$ and ad $a_{1} \cdots$ ada $a_{n}(A) \not \subset P_{n+1}$. If the claim were false, there would be

$$
\operatorname{ad} a_{1} \cdots \operatorname{ad} a_{n}(A) \subset \bigcap_{P \in \mathcal{P}, I_{n} \not P P} P
$$


Let $b_{1} \in A$ such that $b_{2}=\operatorname{ad} a_{1} \cdots$ ad $a_{n}\left(b_{1}\right) \notin P_{n}$ and note that $b_{2} \in \bigcap_{P \in \mathcal{P}, I_{n} \not P} P$. Let $X$ be a finite-dimensional irreducible Banach left $A$-module with $P_{n}=\{a \in A: a X=0\}$. By the Jacobson density theorem it is a simple matter to show that there exists $b_{3} \in A$ such that $\operatorname{dim} b_{3} b_{2} X=1$ and $\left(b_{3} b_{2}\right)^{2} X=0$. Set $c=b_{3} b_{2}$. Since $\operatorname{dim} c X=1$, there exists a linear functional $f$ on $A$ such that $(c a c-f(a) c) X=0$ for all $a \in A$. Consequently, we have

$$
c a c-f(a) c \in I_{n-1} \cap P_{n} \cap\left(\bigcap_{P \in \mathcal{P}, I_{n} \not \subset P} P\right)=\operatorname{Rad}(A)=0 .
$$

Hence $\operatorname{dim} c A c<\infty$. Since $c^{2} \in P_{n}$, we have

$$
c^{2} \in I_{n} \cap\left(\bigcap_{P \in \mathcal{P}, I_{n} \notin P} P\right)=\operatorname{Rad}(A)=0 .
$$

Consequently, $(\operatorname{adc})^{2} a=-2 c a c$ for all $a \in A$. Since $\operatorname{dim}(\operatorname{ad} c)^{2}(A)<\infty$, we see that $D(\operatorname{adc})^{2}$ is continuous. On the other hand, it is immediate that $D(\operatorname{adc})^{2}-(\operatorname{adc} c)^{2} D$ is continuous. Therefore $(\operatorname{adc})^{2} D$ is continuous and hence $(\operatorname{adc})^{2}(\mathcal{S}(D))=0$. Thus $c \mathcal{S}(D) c=0$ and

$$
0=Q_{P_{n}}(c) Q_{P_{n}}(S(D)) Q_{P_{n}}(c)=Q_{P_{n}}(c)\left(A / P_{n}\right) Q_{P_{n}}(c)=0 .
$$

From this it follows that $Q_{P_{n}}(c)=0$, a contradiction. Choose $P_{n+1}$ with the claimed properties. On account of [1, Corollary 1] the linear subspace of $A / P_{n+1}$ generated by $\left\{\operatorname{ad} Q_{P_{n}}(a)\left(Q_{P_{n+1}}(A)\right): a \in A\right\}$ equals $A / P_{n+1}$. Since $Q_{P_{n+1}}\left(I_{n}\right)=A / P_{n+1}$ and ada $\cdots \operatorname{ad} a_{n}(A) \not \subset$ $P_{n+1}$, we conclude that there is $a_{n+1} \in I_{n}$ such that ad $a_{1} \cdots$ ad $a_{n} \operatorname{ad} a_{n+1}(A) \not \subset P_{n+1}$.

Note that, for all $m, n \in \mathbb{N}$,

$$
\begin{aligned}
Q_{P_{n}} D \operatorname{ad} a_{1} \cdots \operatorname{ad} a_{m}= & \sum_{k=1}^{m} \operatorname{ad} Q_{P_{n}}\left(a_{1}\right) \cdots \operatorname{ad} Q_{P_{n}}\left(D\left(a_{k}\right)\right) \cdots \operatorname{ad} Q_{P_{n}}\left(a_{m}\right) \\
& +\operatorname{ad} Q_{P_{n}}\left(a_{1}\right) \cdots \operatorname{ad} Q_{P_{n}}\left(a_{m}\right) Q_{P_{n}} D
\end{aligned}
$$

Since $\sum_{k=1}^{m} \operatorname{ad} Q_{P_{n}}\left(a_{1}\right) \cdots \operatorname{ad} Q_{P_{n}}\left(D\left(a_{k}\right)\right) \cdots \operatorname{ad} Q_{P_{n}}\left(a_{m}\right)$ is continuous and $Q_{P_{n}}\left(a_{m}\right)=0$ if $m>n$, Lemma 1 shows that $Q_{P_{n}} D$ ada $a_{1} \cdots \operatorname{ad} a_{n}$ and therefore $\operatorname{ad} Q_{P_{n}}\left(a_{1}\right) \cdots \operatorname{ad} Q_{P_{n}}\left(a_{n}\right) Q_{P_{n}} D$ are continuous for some $n \in \mathbb{N}$. Accordingly, we have

$$
0=\operatorname{ad} Q_{P_{n}}\left(a_{1}\right) \cdots \operatorname{ad} Q_{P_{n}}\left(a_{n}\right) Q_{P_{n}}(S(D))=\operatorname{ad} Q_{P_{n}}\left(a_{1}\right) \cdots \operatorname{ad} Q_{P_{n}}\left(a_{n}\right)\left(A / P_{n}\right)
$$

which contradicts the choice of $a_{n}$ and $P_{n}$.

Theorem. Let $D$ be a Lie derivation on a semisimple Banach algebra $A$. Then $\mathcal{S}(D) \subset \mathcal{Z}(A)$. Accordingly, $D$ is continuous if $\mathcal{Z}(A)=0$. 
Proof. If $A$ is a complex Banach algebra without unit, then its unitization $A_{1}$ is a unital semisimple complex Banach algebra and we extend $D$ to a Lie derivation on $A_{1}$ by defining $D_{1}(1)=0$. The preceding lemma shows that $\mathcal{S}\left(D_{1}\right) \subset \mathcal{Z}\left(A_{1}\right)$. On the other hand, we have $\mathcal{S}(D) \subset \mathcal{S}\left(D_{1}\right)$ and therefore $\mathcal{S}(D) \subset A \cap \mathcal{Z}\left(A_{1}\right)=\mathcal{Z}(A)$.

If $A$ is a real Banach algebra, then we consider its complexification $A_{\mathbf{c}}$ and we extend $D$ in the obvious way to a Lie derivation $D_{\mathrm{C}}$ of $A_{\mathrm{C}}$. From what has already been proved, we conclude that

$$
\mathcal{S}(D) \subset A \cap \mathcal{S}\left(D_{\mathrm{C}}\right) \subset A \cap \mathcal{Z}\left(A_{\mathrm{C}}\right)=\mathcal{Z}(A)
$$

Next we show a discontinuous derivation on a semisimple Banach algebra whose centre is $\mathbb{C}$.

Example. Let $A$ be the Banach algebra of the Hilbert-Schmidt operators on an infinite-dimensional complex Hilbert space with an identity adjoined. $A$ is semisimple, $\mathcal{Z}(A)=\mathbb{C}$, and $[a, b]$ is a trace class operator whenever $a, b$ lie in $A$. Therefore $[A, A]$ is not closed in $A$ and hence there exists a discontinuous linear functional $f$ on $A$ whose kernel contains $[A, A]$. The discontinuous linear operator $D$ from $A$ into itself defined by $D(a)=f(a) 1$ is easily seen to be a Lie derivation of $A$.

\section{REFERENCES}

1. M. BREŠAR, Commuting traces of biadditive mappings, commutativity preserving mappings and Lie mappings, Trans. Amer. Math. Soc. 335 (1993), 525-546.

2. P. DE LA HARPE, Classical Banach-Lie algebras and Banach Lie groups of operators in Hilbert space (Lecture Notes in Math. 285, Springer-Verlag, Berlin, 1972).

3. I. N. Herstern, Lie and Jordan structures in simple, associative rings, Bull. Amer. Math. Soc. 67 (1961), 517-531.

4. B. E. Johnson and A. M. Sinclair, Continuity of derivations and a problem of Kaplansky, Amer. J. Math. 90 (1968), 1067-1073.

5. W. S. Martindale, 3rd, Lie derivations of primitive rings, Michigan Math. J. 11 (1964), 183-187.

6. W. S. Martindale, 3rd, Lie isomorphisms of prime rings, Trans. Amer. Math. Soc. 142 (1969), 437-455.

7. C. R. MIERs, Lie derivations of von Neumann algebras, Duke Math. J. 40 (1973), 403-409.

8. C. R. Miers, Lie triple derivations of von Neumann algebras, Proc. Amer. Math. Soc. 71 (1978), 57-61. 
9. T. W. PALmer, Banach Algebras and the General Theory of *-algebras. Volume I: Algebras and Banach Algebras (Cambridge University Press, 1994).

10. M. P. Thomas, Primitive derivations on non-commutative Banach algebras, Pacific $J$. Math. 159 (1993), 139-152.

Departamento de Analisis Matematico

FaCultad de Ciencias

UNIVERSIDAD DE GRANADA

18071 GraNADA

SPAIN

E-mail: avillena@goliat.ugr.es 